\title{
Relations between complexity, quality and cognitive automation in mixed-model assembly
}

\author{
Å. Fast-Berglund ${ }^{\mathrm{a}, *}$, T. Fässberg ${ }^{\mathrm{a}}$, F. Hellman ${ }^{\mathrm{b}}$, A. Davidsson $^{\mathrm{c}}$, J. Stahre ${ }^{\mathrm{a}}$ \\ a Department of Product and Production Development, Chalmers University of Technology, Hörsalsvägen 7, SE-41296 Gothenburg, Sweden \\ ${ }^{b}$ Volvo Truck Corporation, Gothenburg, Sweden \\ c Volvo Cars Corporation, Gothenburg, Sweden
}

\section{A R T I C L E I N F O}

\section{Article history:}

Received 14 September 2012

Received in revised form 28 March 2013

Accepted 11 April 2013

Available online $\mathrm{xxx}$

\section{Keywords:}

Assembly

Complexity

Quality

Cognitive automation

\begin{abstract}
A B S T R A C T
Modern assembly systems are adapting to the increased mass customization resulting in shorter cycle times, more variants and increased complexity for final assembly operators. More variants require additional support functions targeted for assembly operators to avoid assembly errors. An industrial case study was carried out in order to examine the relations between complexity, quality and cognitive automation. Complexity was quantified by the measure Operator Choice Complexity (OCC). Assembly errors were measured from historical data and cognitive automation was assessed on a task level. Results show that complexity positively correlates with assembly errors and that the usage of cognitive support is low. Over $60 \%$ of all tasks in the study were performed based on own experience. Increased usage of cognitive automation is needed in final assembly to minimize the negative effects of complexity.
\end{abstract}

(C) 2013 The Society of Manufacturing Engineers. Published by Elsevier Ltd. All rights reserved.

\section{Introduction}

Increasing complexity is one of the main challenges for production companies [1] and complexity is closely related to the increased number of product variants induced by mass customization. The future holds a more customized market and mass customization has been recognized as the new paradigm for production [2,3]. In the automotive industry, customization is achieved by offering the customer extensive amount of options to choose from. In order to handle the variety in a final assembly context, mixed-model assembly lines are being used. This approach makes it possible to produce different customized models on one assembly line, which results in unique products but also a more complex work environment for the assembly operators. Numerous product variants increase the amount of information to and from the assembly personnel since information regarding each product variant must be available. Previous research has shown that the amount and content of information are contributors to production complexity [4,5]. In an assembly environment, new and effective information flows are required to handle the vast amount of information available due to the high variety of products and parts. Also, the amount of information needed by the operators is individual

\footnotetext{
* Corresponding author. Tel.: +46 317723686.

E-mail addresses: asa.fasth@chalmers.se (Å. Fast-Berglund), tommy.fassberg@chalmers.se (T. Fässberg), filip.hellman@volvo.com (F. Hellman), adavid12@volvocars.com (A. Davidsson), johan.stahre@chalmers.se (J. Stahre).
}

and dependent on their level of expertise [6]. Product variation driven by mass customization creates a vast need for information to support operators working in final assembly. More variants and parts to handle means more decision making, and therefore better decision support is required for the assembly operators. In an assembly context automated solutions no longer only consider mechanical tasks; they also concern cognitive support for information and control tasks. A transition has been made from tools such as electric screwdriver (that only provided mechanical assistance) to "smart" tools which also provide cognitive support [7]. Task complexity and perceived complexity might be targeted by the use of cognitive automation for the operator e.g. technical support to know what and how to assemble. Better support is important as human cognitive skills are increasingly crucial when manufacturing systems are becoming more complex and subjected to changes and uncertainties [8]. In order to optimize a system, some kind of allocation has to be done. There are different allocation approaches that are used in different stages and at different levels at companies:

Task allocation is usually made later, often during system implementation [9]. This type of allocation is often a static allocation based on global optimization [10].

Resource allocation or product/resource mapping means that one or more possible resources are identified for each operation. The desired degree of flexibility will decide how many alternative resources that is included in this resource allocation and a final choice has to be determined, e.g. by optimization [11]. To be able to reduce complexity and to maintain routing flexibility in a system 
resource allocation and cognitive support to these resources is believed to be a good solution for mixed-model assembly systems [12].

Based on the introduction and earlier empirical findings, our hypothesis is:

Cognitive support offered to the operators' individual needs could make the resource allocation of the stations in a complex mixedmodel assembly line easier, without decreasing the performance of the work.

An empirical study has been conducted in order to find out if there are any correlation between the area of complexity, cognitive automation and performance (in this case Quality). In this paper only quantitative methods are used in order to test if this hypothesis could be supported only by those kinds of methods or if other more qualitative methods are needed as well to get a broader picture of the problem.

\section{Parameters of interest}

In order to answer the hypotheses some area of interest has been chosen and also some delimitations within these areas has been done. A more detailed description of the areas is presented in the following sections.

\subsection{Complexity}

Complexity has been defined in several ways, Weaver [13] for instance, describes complexity as the degree of difficulty to predict the system properties, given the properties of the systems parts. The relationship between complexity and variety of products and parts has been investigated by several authors [14-16] and has been referred to as the main cause of complexity within the automotive industry [15]. To meet requirements from mass customization, many assembly systems are using a mixed-model assembly approach as an enabler for the high variety of products. Although mixed model assembly facilitates a high variety, such systems tend to become more complex as variety increase [17]. The complexity ranges from planning aspects on a managerial level down to increased assembly choices on an operator level. In order to understand operators perceived complexity, a holistic view of complexity (including subjective complexity) is of importance [18]. Complexity could be divided into two main areas; objective complexity and subjective complexity:

Objective complexity can be further divided into:

- Structural complexity [19] or static complexity [20]. Characteristics are related to fixed nature of products, hierarchical structures, processes, variety, and strength of interactions.

Within the area of objective complexity could also be in counted task optimization and sequence planning of operations in order to reduce the complexity $[11,12,21,22]$.

Subjective production complexity, the same production system or situation may be perceived differently depending on a number of different factors such as individuals' skills, competence and experience [17]

- Behavioral complexity [23] is characterized by dynamism, nonlinearity, deviation from equilibrium, history, adaptive, emergent structures, and self-organization evolution.

- Dynamic complexity, which is caused by external and internal sources within the operation, like variations in dates and amounts due to material shortness, breakdowns, insufficient supplier reliability.

In order to answer the hypotheses, this paper will only discuss objective complexity and in short the dynamic complexity of the system, as parameters of complexity.

\subsection{Cognitive automation}

In production, especially in an assembly context, cognitive automation is aimed at supporting decision-making ensuring that error-free products are produced. An increased cognitive Level of Automation (LoA) (i.e. more decision-making tasks are performed automatically) could improve the operators' work situation and decrease their workload while the same physical automation is maintained [24]. This encourages a shift in focus from physical to cognitive automation. One definition of cognitive automation has been provided by Thurman et al. [25] "Cognitive automation is software intended to automate cognitive activities, such as situation assessment, monitoring, and fault management, that are currently performed by human operators". Automation has an impact on the operators cognitive functions, his/her thinking as well as doing [26]. Frohm [27] has divided both physical and cognitive automation in his LoA taxonomy ranging totally manual to fully automatic visualized in Fig. 1.

In the visualized matrix three areas are presented: human assembling and monitoring, machine/technique monitoring, and machine assembling. At an automotive final assembly line a majority of tasks are performed by humans and are in the area of human assembling and monitoring. In this area, cognitive automation ( LoA $\left._{\text {cognitive }}\right)$ could be described as the amount of technique and information provided to the operator in order to know what, how and when to do a specific task in the most efficient way. When a tool or machine is performing the task i.e. higher physical automation ( LoA $\left._{\text {Physical }}=5-7\right)$, the cognitive automation is mainly used for control and supervision $\left(\operatorname{LoA}_{\text {Cognitive }}=4-7\right)$ [28].

The matrix will be used as the main part in the cognitive automation measures in the empirical study.

\section{Case company and selected area}

This section describes shortly the problem area and why the company and the particular area has been chosen to test the hypothesis.

Volvo Car Corporation (VCC) manufactures approximately 400,000 cars per year, with the two main assembly plants located in Gent, Belgium and in Torslanda, Sweden. At the Torslanda plant five models based on three platforms; V70, XC70, S80, XC90 and V60 are produced with a total volume of 136,323 cars in 2010 . One serial flow mixed-model assembly line is used for all five models in the final assembly plant. The assembly line is divided into different line segments allowing buffers in between. A driven conveyor line continuously paces a majority of the assembly line segments. Short cycle time and a high division of work characterize the assembly. The empirical study was carried out at the end of 2011, and then, the tact time was $66 \mathrm{~s}$, with a variation between the line segments. Subassembly lines were to some extent used to supply the main assembly line with parts. The line segment was chosen by the company due to the high complexity. The complexity contained of high number of product variants and a large number of parts at the line. The chosen area consisted of sixteen stations, however, only seven were included in the study (visualized with by gray operators in Fig. 2). The chosen stations were a part of a pre-assembly area for a preparation line of engines. At the selected line, engines were customized to fit customer demands. The assembled engines were 


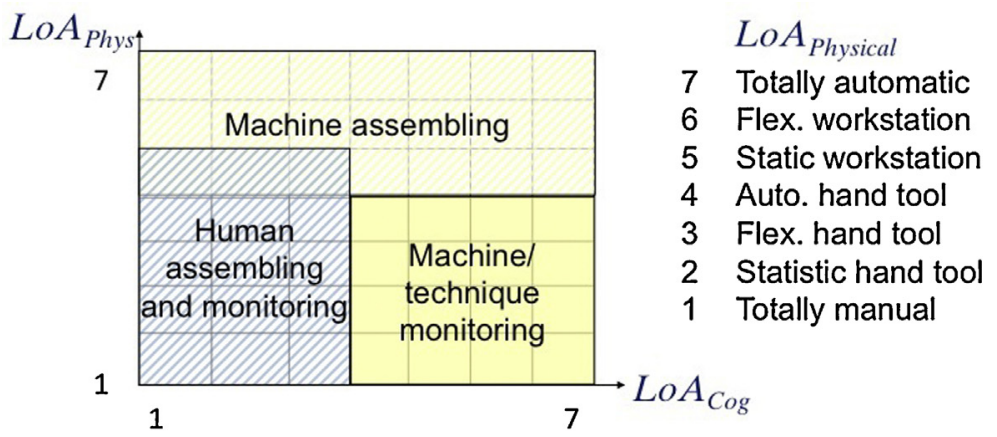
Lo $A_{\text {Cognitive }}$
Totally automatic
Intervene
Supervising
Questioning
Teaching
Decision giving
Totally manual

Fig. 1. LoA matrix [28] based on Frohm's taxonomy [27].

Team Area 1

Team Area 2

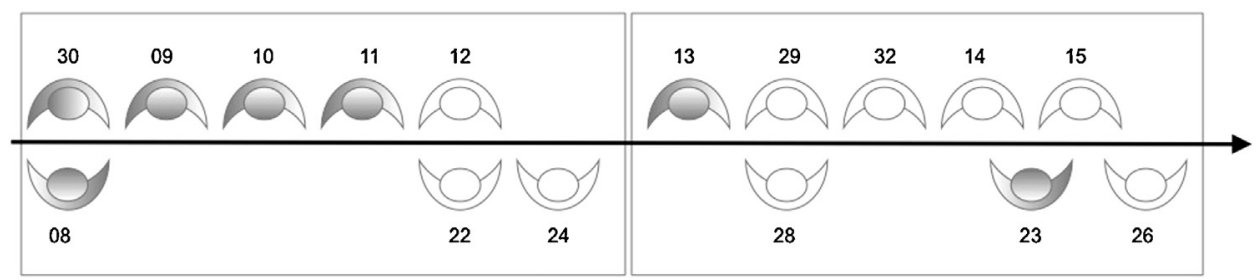

Fig. 2. Selected area, total number of stations and selected stations.

used to supply all models and variants on the main assembly line. There were three areas for the pre-assembly of the engines and this is the second area named Power Pack 2 (PP2).

The pre-assembly area was organized as a serial flow assembly line without buffers between the stations. A driven assembly line conveyor paced the line and the assembly line was characterized by short tact times. In this study two teams were involved, each team was responsible for 6-8 stations. There was one team leader within each team responsible for error reporting and other support functions. There was one operator working at each station and work rotations between the stations were done regularly.

\section{Design of the experiment: methods used}

Three different types of methods have been chosen to show the relation between complexity, quality and cognitive support to the operators. The data collected for the three methods has been collected from VCCs data system (i.e. assembly errors and data for the OCC measurement), by observations at the line (for the measure of cognitive automation) and by semi-structured interviews. In order to use only quantitative methods some parts of the methods used is limited to only use the quantitative part of the methods. This is mostly true for analyzing the cognitive automation and the DYNAMO++ methodology which consists of both qualitative and quantitative collection of information. According to [29] this has its pros and cons; qualitative data is more 'rich', time consuming, and less able to be generalized, while quantitative data is more efficient, able to test hypotheses, but may miss contextual detail, which will be brought up in the discussion.

The methods are also chosen due to the fact that they are useable in the running phase of a manufacturing system, the data is collected at station and task level and the personnel involved in collecting and explaining the data are production engineers, operators or other persons closely related to the value added tasks.

\subsection{Operator Choice Complexity}

The method used for collecting data about the complexity of the seven stations defined as "operators choice complexity" [11] and concerns all choices that the assembly operator can make and the risk for error associated with these choices. OCC is a measure developed to quantify human performance in making choices [13]. The measure is based on an entropy function and is defined: "Choice complexity is the average uncertainty or randomness in a choice process, which can be described by a function $H$ in the following form".

$H i(\rho i 1, \rho i 2, \ldots, \rho i M i)=-C \cdot \sum_{j=1}^{M i} \rho i j \cdot \log \rho i j$

In Eq. (1), $\rho i j$ is the occurrence probability of a state $j$ in the random process $i, j \in[1 \mathrm{Mi}]$. $C$ is a constant depending on the base of the logarithm function chosen, if $\log 2$ is selected, $C=1$ [11].

By assessing the number of possible variants at a given assembly station and the given demand for each variant, the probability for each variant is given. This probability is then used to get a complexity measure for the station is given based on the variance. This reasoning can be expanded to include choices of fixtures, tools and parts. Furthermore, the OCC could also be used to see how much of the complexity that is transferred to the up-stream stations and how much of the complexity that is added on that particular station i.e. feed complexity [22]. This is of importance when choosing the cognitive support at the station.

OCC was calculated for each of the seven selected stations. Input to the equation was the number of variants that occurs at each station and the demand for each variant based on 3835 cars produced during one week. The probability $\rho$ was calculated for each variant $j$ and for each station $i$ and the total Operator Choice Complexity was calculated with Eq. (1), the unit scale in of the measure is bit.

\subsection{Operator performance}

The operators' performance was measured in terms of quality or assembly errors. The data were collected by extracting errors reported to the internal quality system named ATACQ. When reported, assembly errors were classified into different error categories e.g. "not connected" and "incorrectly fitted". Errors were extracted for a time period of 16 weeks for the seven stations 


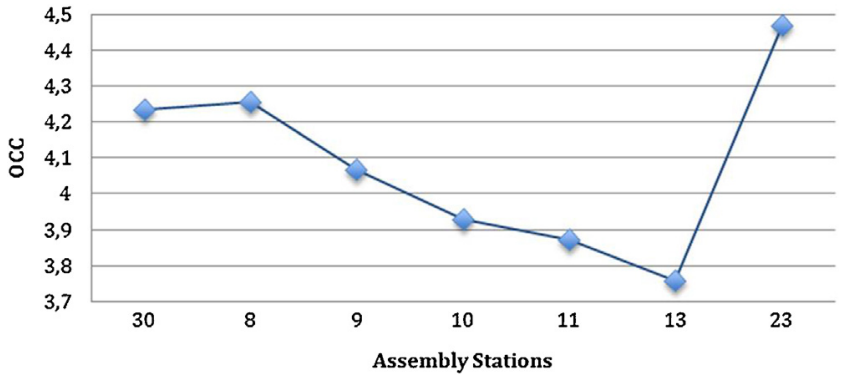

Fig. 3. Distribution of OCC per station based 3835 produced cars.

included in the study. The errors caused by material and parts defects were excluded prior to the study i.e. only assembly errors were included in the study.

Assembly errors were discovered either by the operator performing the task, a quality controller, at a Standardized Inspection Point (SIP) or by an automatic system such as a vision camera. Reported errors were associated with the product architecture, allowing for errors to be traced back to the station and operation where it occurred.

To analyze the relation between assembly errors and OCC, bivariate correlation analysis was carried out in order to evaluate the relationship between the parameters OCC and assembly errors. Statistical analyses were performed with JMP 9.0.0. ${ }^{1}$

\subsection{Cognitive automation}

The cognitive automation is measured on a task level, using the reference matrix in Fig. 1 (there are additional descriptions of each level in terms of examples of tools or support from previous studies). The matrix is included in a methodology called DYNAMO++, which aims to provide a structured approach toward task allocation and resource allocation in final assembly systems [18]. In the measurement phase of the DYNAMO++ methodology a LoA assessment method is used to visualize the current LoA for one or several operations of interest.

The LoA measure was made from direct observations at the stations and from collecting descriptions from SOP (Standardized Operations Procedure) sheets and WES (work element sheets). Two models were assessed for each station, the most common model regarding demand and the heaviest model to produce regarding time i.e. the most complex and the less complex product.

\section{Results}

This section will present the results from each method used. The results will then be discussed in relation to each other in Section 6.

\subsection{Operator Choice Complexity}

The result of the OCC measure for the seven stations is visualized in Fig. 3. The mean value of the OCC was 4.1. The lowest value (3.8) was identified at station 13 while the highest value (4.5) was observed at station 23 . Thus indicating that station 23 was the most complex and station 11 the least complex.

Assembly personnel considered the main causes of complexity as the amount of tasks to be performed within a limited timeframe and workspace. Complexity issues discussed by assembly operators were uneven work pace caused by balancing problems due to the high number of variants and the need to remember how to assem-

\footnotetext{
1 http://www.jmp.com/.
}

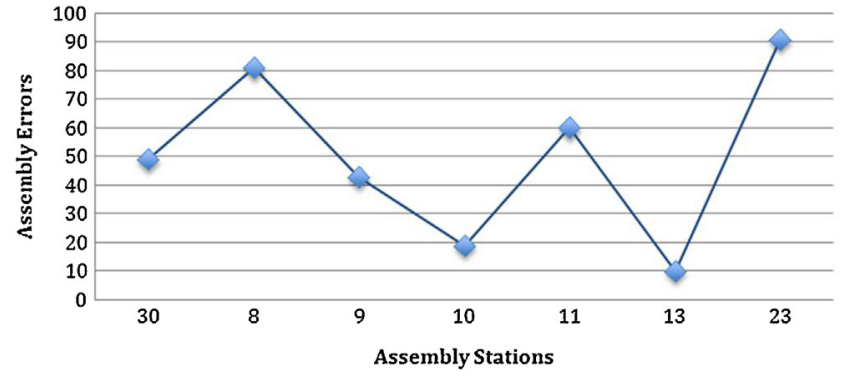

Fig. 4. Distribution of assembly errors per station for a time period of 16 weeks.

Table 1

Assembly errors per classification type.

\begin{tabular}{lcc}
\hline Error type & Number of errors & Percentage of total errors \\
\hline Not connected & 106 & $30 \%$ \\
Incorrectly fitted & 82 & $23 \%$ \\
Missing & 51 & $14 \%$ \\
Not tightened & 38 & $11 \%$ \\
Loose & 29 & $8 \%$ \\
Disassembly & 28 & $8 \%$ \\
Broken & 8 & $2 \%$ \\
Wrong type & 7 & $2 \%$ \\
Other & 4 & $1 \%$ \\
Total & 353 & $100 \%$ \\
\hline
\end{tabular}

ble different or unusual variants. Complexity was related to issues of variants, uncertainties, work content and time pressure. Furthermore, in station 23 there were an increase in feed complexity due to a new tool and new components that the operator needed to learn.

"We have a lot of parts besides the AC to put in place/.../you have to work faster for a short period of time then."

Assembly Operator A, regarding station 23 .

\subsection{Assembly errors}

A total of 534 errors were found in the quality system ATACQ distributed over the studied stations. Due to part defects originated from suppliers, 181 errors were excluded, leaving a total of 353 assembly errors. The distribution of errors can be found in Fig. 4. The mean value of errors was 50 . Station 13 had the least amount of errors (9), while station 23 had the most (91).

Assembly errors were categorized in eleven categories, were the top four categories accounted for $79 \%$ of the total number of errors. These categories were associated with errors where parts had not been properly connected, incorrectly assembled, missing parts, or incorrectly tightened. Common errors included loose parts e.g. plastic covers not having been dismantled. The characteristics for a majority of the measured errors are visualized in Table 1.

\subsection{Cognitive automation}

A total of 269 assembly tasks were measured within the seven stations, divided into low complexity (127 tasks) and high complexity (142 tasks) product, as illustrated in Fig. 5. As seen the high complexity product has approximately $10 \%$ tasks to be performed at the line, which could contribute to the high complexity of the product. This will also result in a higher OCC measure, discussed in Section 6.3.

LoA measurements reveal that $62 \%$ of all task for the high complexity product were performed with LoA $_{\text {Physical }}=1$ and Lo $_{\text {Cognitive }}=1$ i.e. by hand and with own experience. For the low complexity variant it was $64 \%$. The high percentage of tasks 
Low Complexity variant

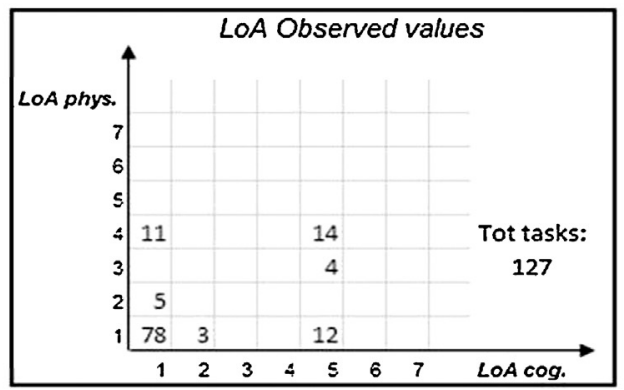

High Complexity variant

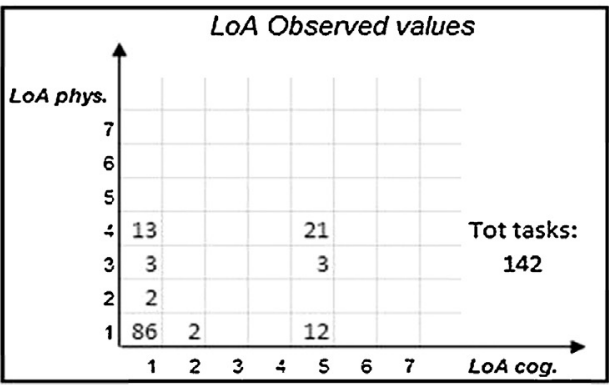

Fig. 5. LoA measurement for low and high complexity product.

performed without cognitive support could have had an impact on quality.

In contrast, $25 \%$ of the tasks for the high complexity product and $24 \%$ for the low complexity product were performed with a $\operatorname{LoA}_{\text {cognitive }}=5$ i.e. supervising. The supervision was mainly accomplished by Pick-By-Light (PBL) systems guiding the assembly operators, which parts or bits to choose as illustrated in Fig. 6.

"Good support, you know what to pick. ... It had worked without, but it would have taken a longer time."

Assembly Operator B, regarding Pick By Lights

The use of PBL ranged from 0 light at station 10-15 lights at station 8. The implementation of light was based on the risk of committing errors. If a risk for confusion of parts was assessed a PBL or another poka-yoke solution were suggested.

In addition to mentioned support systems operators were supported in their work by screens, showing information about the sequence of nuts and bolt to tightening and the status of the task. The operators were also provided with feedback from the Pick-By-Lights and haptic feedback from some of the tools. Operator instruction sheets were available at every team area gathered in binders. Parts were presented to the operators in the material facade in bulk packages or by sequence racks assessed as Lo $\mathrm{A}_{\text {cognitive }}=2$ = working order.

\section{Analysing the result: relations between the three areas}

Three parameters have been measured; OCC, Assembly errors and cognitive automation. The relations of theses parameters have been further investigated as visualized in Fig. 7.

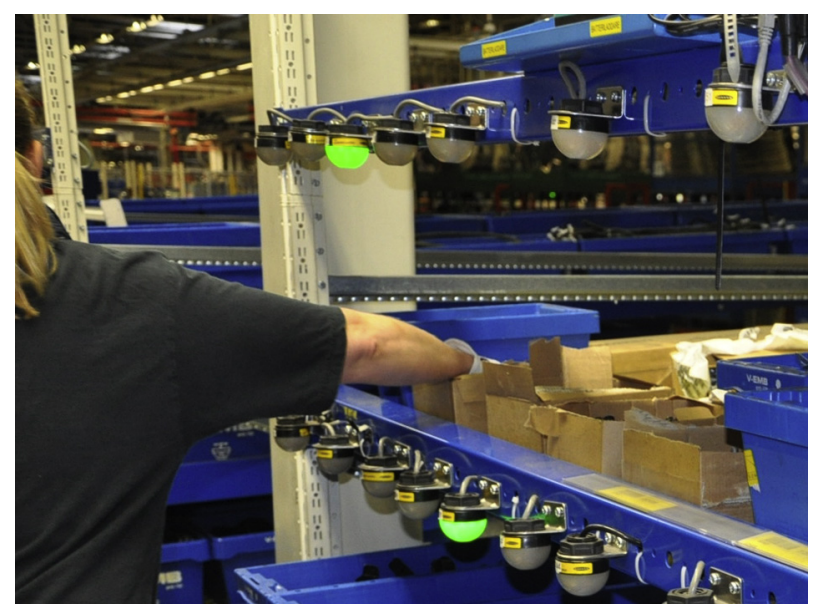

Fig. 6. Pick-By-Light, an example of cognitive automation, $\operatorname{LoA}_{\text {cognitive }}=5$.

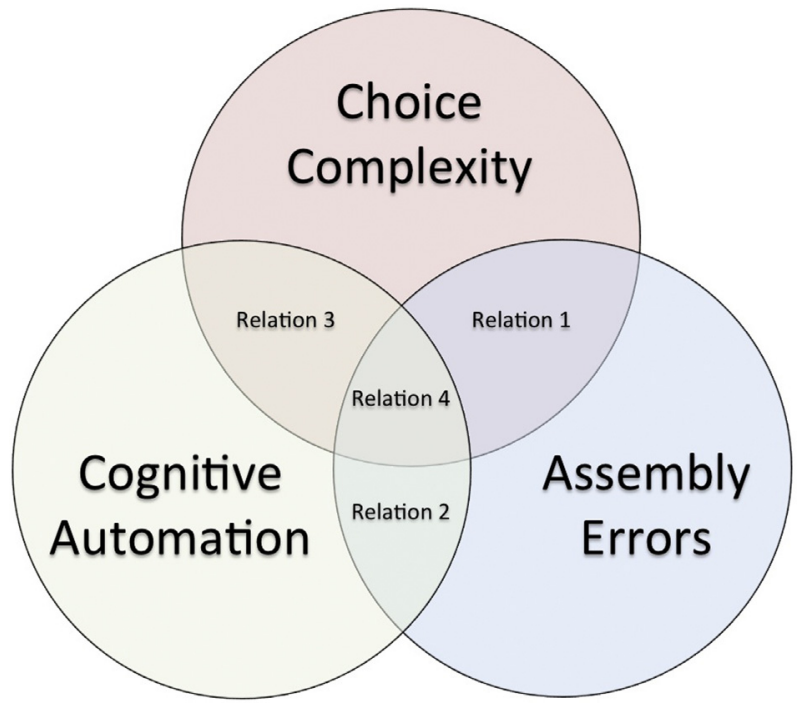

Fig. 7. Overview of the three investigated areas.

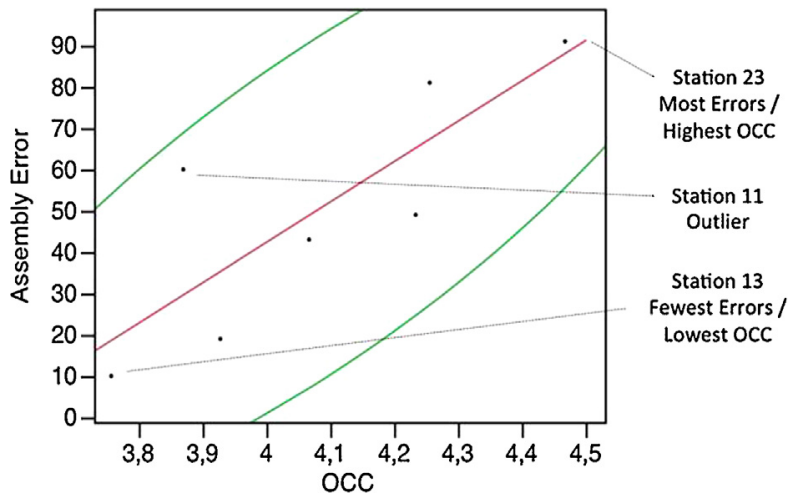

Fig. 8. Relation between Operator Choice Complexity and assembly errors.

\subsection{Relation 1; between Operator Choice Complexity and assembly errors}

When examining the relation between OCC and assembly errors it is evident that a positive linear correlation exists between the two parameters as illustrated in Fig. 8.

As seen in Table 2 the correlation coefficient was 0.819 showing a significant positive correlation between OCC and assembly errors, meaning that an increase of OCC results in more assembly errors.

The additional relations will focus on three stations ${ }^{*}$, the station with the lowest complexity and the lowest number of assembly 
Table 2

Statistical data for two variables OCC and assembly errors.

\begin{tabular}{lrrlll}
\hline Variable & Mean & Std dev & Correlation & Signif. prob & Number \\
\hline OCC & 4.083 & 0.251 & 0.819 & $0.024^{*}$ & 7 \\
Assembly errors & 50.428 & 29.865 & & & \\
\hline
\end{tabular}

errors (station 13), the station with the highest complexity and the highest error rates (station 23) and station 11 which deviated the most from the linear correlation.

\subsection{Relation 2; between assembly errors and cognitive automation}

At station 11 a total of 60 assembly errors were measured during the investigated time period. A total of $63 \%$ (38) of the errors were classified as "not connected" meaning that a signal cable had not been connected. One specific part and task accounted for 38\% (23) of the total errors. The LoA of this specific task was LoA $A_{\text {Cognitive }}=1$ and Lo $A_{\text {Physical }}=1$, meaning that the operation was performed without any support.

"And I can admit I do not know exactly how you are supposed to build, but I build what I regard as optimal."

Assembly Operator A, regarding standardized work

A total of 91 assembly errors were measured and 60\% (54) of the errors were classified as "incorrectly fitted" in station 23 . One single part and task accounted for $51 \%$ (47) of the total errors. The part was either placed in wrong position or missing. The LoA of this specific task was LoA $A_{\text {Cognitive }}=1$ and Lo $A_{\text {Physical }}=1$, meaning that the operation was performed without any support.

In station 13 a total of 10 assembly errors were measured during the investigated time period, classified as "not connected". The low error rate at this station could be explained by that most operations at the station were associated with a high LoA. Part assurance was made with a hand scanner and tightening operations were counted by the system to match the number of tasks planned for.

"I do not know if I would like lights everywhere/. . ./but totally without then you would have to be more alert and you know sometimes when you are stressed you forget."

Assembly Operator A, regarding Pick By Lights

The most common error types for the investigated stations (11, 13 and 23) were "incorrectly fitted" and "not connected". Thus, implying that there is a need for more cognitive support at these stations, especially for cable insertions. Distributions of the most common error types for the three stations are shown in Table 3.

At the investigated stations decision support was given by PBL and information about sequence was given by monitors connected to electrical screwdrivers. Screw driving tasks are easy to control and restrict while manual assembly operation are difficult to monitor and control. Many manual tasks at the stations were to connect electrical cables. However, no support was given when performing these tasks. If a manually performed task were incorrectly performed or missed the error was not acknowledged until later control stations while screw driving operations were controlled within the station boundaries by a control system connected to the tools.

\subsection{Relation 3; between Operator Choice Complexity and cognitive automation}

As seen in Fig. 5, the high complexity product has more tasks to be performed at the line which increases the OCC and also the balancing so that the operators have less time for this product if the pace should remain the same. This means that the feed complexity increases at some stations when the more complex product will be assembled. This could be reduced be adding more smart tools and instruction to these particular stations.

Restrictive automation solutions at a cognitive level from 5 to 7 would minimize a risk of errors due to choice complexity. Support given by cognitive automation can reduce negative effects of increased assembly errors caused by complexity. Furthermore, smart automation in terms of fixtures and sensors on the hand-tools will also decrease the assembly errors.

\subsection{Relation 4; between cognitive automation, Operator Choice Complexity and assembly errors}

Results from previous sections illustrate that complexity, quality, and cognitive automation are related. A significant positive correlation between OCC and assembly errors was observed. Cognitive automation was used for many assembly tasks both for supervision and guidance. However, more than $60 \%$ of the assembly tasks lacked cognitive support and were performed by own experience i.e. no use of cognitive automation, implying that there is a need for new automation solutions. According to Reason [30] "We cannot change the human condition, but we can change the conditions under which humans work." One way to create a change of work conditions is by using cognitive automation to support assembly operators. Earlier empirical results [31] show that in general, system complexity, does affect performance negatively and that training and that man/machine interface plays important roles in minimizing the negative effect of system complexity on performance. By increase the usage of cognitive automation the negative effects of choice complexity in terms of quality can be reduced.

\section{Discussion and conclusion, hypotheses due to earlier tests}

The result of this study shows that it is possible to use quantitative measures in order to show relation between complexity, quality and cognitive automation. A significant positive correlation was observed between OCC and assembly errors meaning that increased choices lead to more assembly mistakes. Several examples of cognitive support were identified although over $60 \%$ of all tasks were performed only relying on own experience. The classification of assembly errors showed that errors were more common for task performed manually and without any cognitive support. Cognitive automation can be used to reduce the negative effects of increased choices thus reduce the negative effects of complexity. Increased and more precise usage of cognitive automation targeting

Table 3

Summary of errors at station 11,23 and 33 .

\begin{tabular}{|c|c|c|c|c|}
\hline Error Code & Errors (station 11) & Errors (station 13) & Errors (station 23) & Total of Errors \\
\hline Incorrectly fitted & 4 & - & 54 & 58 \\
\hline Not Connected & 38 & 10 & 5 & 53 \\
\hline Not Tightened & 2 & - & 19 & 21 \\
\hline Missing & 14 & - & 5 & 19 \\
\hline Total & 60 & 10 & 91 & 161 \\
\hline
\end{tabular}


stations with high complexity can reduce assembly errors in final assembly.

Based on the empirical findings, some answers could be done to the hypothesis;

Cognitive support is needed, and should be based on the operators needs but also on the transferring and feeding complexity at each station.

A believe is that with this support the performance i.e. quality will increase.

However in order to get the full picture about the cognitive support to the operators, further research and empirical studies has to be made due to perceived complexity among the operators. This because we believe that it is not enough to just investigate the objective complexity by using quantitative methods, but to also consider the operators own view of the assembly line in order to design the right cognitive support for them. Furthermore, a more detailed planning and scheduling of the transferred and feeding complexity is needed in order to balance the high complex product between the stations.

\section{Acknowledgments}

The authors like to express their gratitude to all involved personnel at VCC and to the collages within the COMPLEX project. This work has been funded by Vinnova within the program Production Strategies and Models for Product Realization. This work has been carried out within the Sustainable Production Initiative and the Production Area of Advance at Chalmers. The support is gratefully acknowledged.

\section{References}

[1] ElMaraghy W, ElMaraghy H, Tomiyama T, Monostori L. Complexity in engineering design and manufacturing. CIRP Annals - Manufacturing Technology 2012;61:793-814

[2] Pine BJ. Mass Customization: The New Frontier in Business Competition. Boston, MA: Harvard Business School Press; 1993.

[3] Jovane F, Koren Y, Boer CR. Present and future of flexible automation: towards new paradigms. CIRP Annals - Manufacturing Technology 2003;52:543-60.

[4] ElMaraghy WH, Urbancic RJ. Assessment of manufacturing operational complexity. CIRP Annals - Manufacturing Technology 2004;53:401-6.

[5] Urbanic RJ, ElMaraghy WH. Modeling of manufacturing process complexity. In: ElMaraghy HA, ElMaraghy WH, editors. Advances in design. London: Springer; 2006. p. 425-36.

[6] Fjällström S, Säfsten K, Harlin U, Stahre J. Information enabling efficient production ramp-up. Journal of Manufacturing Technology Management 2009;20:178-96.

[7] Fasth Å, Mattsson S, Fässberg T, Stahre J, Höög S, Sterner M, et al. Development of production cells with regard to physical and cognitive automation - a decade of evolution. In: International symposium on assembly and manufacturing (ISAM 11). 2011.

[8] Grote G. A participatory approach to the complementary design of highly automated work systems. In: Bradley GE, editor. Human factors in organizational design and management IV, Amsterdam. 1994. p. 115-20.

[9] Waterson PE, Gray MTO, Clegg CW. A sociotechnical method for designing work systems. Human Factors 2002;44:376.

[10] Valle CD, Camacho EF, Toro M. A model for assembly sequence planning in a multirobot environment. In: Proceeding of the 15th IFAC world congress. 2002.

[11] Lennartson B, Bengtsson K, Yuan C, Andersson K, Fabian M, Falkman P, et al. Sequence planning for integrated product, process and automation design. IEEE Transactions on Automation Science and Engineering 2010;7.

[12] Fasth Å, Provost J, Fabian M, Stahre J, Lennartson B. From task allocation towards resource allocation when optimising assembly systems. Procedia CIRP 2012;3:400-5.

[13] Weaver W. Science and complexity. American Scientist 1948;36:536.

[14] MacDuffie JP, Sethurman K, Fisher ML. Product variety and manufacturing performance: evidence from the international automotive assembly plant study. Management Science 1996;42:350-69.
[15] Schleich H, Schaffer L, Scavarda F. Managing complexity in automotive production. In: 19th international conference on production research. 2007.

[16] Hu SJ, Zhu X, Wang H, Koren Y. Product variety and manufacturing complexity in assembly systems and supply chains. CIRP Annals - Manufacturing Technology 2008;57:45-8

[17] Gullander P, Davidsson A, Dencker K, Fasth Å, Fässberg T, Harlin U, et al. Towards a production complexity model that supports operation, re-balancing and man-hour planning. In: The 4th Swedish production symposium (SPS) 2011.

[18] Zhu X, Hu SJ, Koren Y, Marin SP. Modeling of manufacturing complexity in mixed-model assembly lines. Journal of Manufacturing Science and Engineering 2008; 130 .

[19] T. Blecker, W. Kersten, C.M. Meyer, Development of an approach for analyzing supply chain complexity. In: Hamburg Univ. of Technol., MPRA Paper \#5284; 2005, http://mpra.ub.unimuenchen.de/5284/

[20] Frizelle G, Suhov YM. An entropic measurement of queueing behaviour in a class of manufacturing operations. Proceedings of the Royal Society of London 2001;457:1579-601.

[21] Provost J, Fabian M, Lennartson B, Fasth Å, Stahre J. Planning in assembly systems - a common modeling for products and resources. In: ETFA 2012 IEEE international conference on emerging technology and factory automation. 2012.

[22] Zhu X, Hu SJ, Koren Y, Huang N. A complexity model for sequence planning in mixed-model assembly lines. Journal of Manufacturing Systems 2011;31:121-30.

[23] Asan SS. A methodology based on theory of constraints' thinking processes for managing complexity in the supply chain. Germany: Univ. Berlin; 2009.

[24] Fasth Å, Stahre J. Concept model towards optimising Levels of Automation (LoA) in assembly systems. In: Proceedings of the 3rd CIRP conference on assembly technologies and systems. 2010.

[25] Thurman AD, Brann MD, Mitchell MC. An architecture to support incrementa automation of complex systems. In: Proceedings of the 1997 IEEE international conference on systems, man, and cybernetics. 1997.

[26] Hollnagel E. Cognitive functions and automation: principles of human-centered automation. In: Symbiosis of human and artifact. 1995. p. 971-6.

[27] Frohm J, Lindström V, Winroth M, Stahre J. Levels of automation in manufacturing. Ergonomia IJE\&HF 2008;30(3).

[28] Fasth Å, Stahre J, Dencker K. Measuring and analysing Levels of Automation in an assembly system. In: Proceedings of the 41 st CIRP conference on manufacturing systems. 2008 .

[29] Miles, Huberman. Qualitative data analysis; 1994.

[30] Reason J. Human error: models and management. British Medical Journal 2000;320:768-70.

[31] Guimaraes T, Martensson N, Stahre J, Igbaria M. Empirically testing the impact of manufacturing system complexity on performance. International Journal of Operations and Production Management 1999;19:1254-69.

Åsa Fasth, Assistant Professor, who received her PhD in 2012 from Chalmers University of Technology, Gothenburg Sweden. Åsas research focus on automation and Levels of Automation in production and assembly systems and how to allocate tasks between humans and technology in a structured way to achieve competitive benefits.

Tommy Fässberg received his Licentiate degree in 2012. His focus in the thesis was to classify carrier and content of information in order to improve the information support solution for the operators in a complex production.

Filip Hellman holds a BSc in Mechanical Engineering and a MSc in Production Engineering from Chalmers University of Technology in Gothenburg, Sweden. He has experience in manufacturing systems from Volvo Car Corporation, Volvo Construction Equipment and Volvo Truck Corporation. He is currently employed at Volvo Truck Corporation, Group Trucks Operations. In his current position he develops methods and systems within the areas of assembly line balancing, flow simulation and layout within the virtual manufacturing domain.

Dr. Anna Davidsson received her PhD in 1999 from Chalmers University of Technology in Gothenburg, Sweden. She is currently working at Volvo Cars Corporation. In her current position she works with manufacturing research projects and serves as a link between research and industry. Her special focus area lies in Management of Production and Humans in Production.

Johan Stahre is Chair Professor of Production Systems at Chalmers University of Technology in Gothenburg, Sweden and head of the Production Systems Division. Prof. Stahre has a MSc from Linköping University and received his PhD from Chalmers in 1995. His research focus is on manufacturing automation, humanautomation interaction, and human supervisory control of production systems. He is former Chairman and co-founder of the Swedish Production Academy and academic coordinator for the Swedish node of ManuFuture, the European Technology Platform for Manufacturing. 